\title{
Biomass Derived Activated Carbon/Epoxy Composite as Microwave Absorbing Material
}

Praveen Negi

National Institute of Technology Kurukshetra

Ashavani Kumar ( $\nabla$ ashavani@yahoo.com )

National Institute of Technology Kurukshetra https://orcid.org/0000-0002-1192-1724

\section{Research Article}

Keywords: Activated carbon, microwave absorbing material, biomass, composite materials, reflection loss

Posted Date: February 18th, 2021

DOI: https://doi.org/10.21203/rs.3.rs-164621/v1

License: (c) (i) This work is licensed under a Creative Commons Attribution 4.0 International License. Read Full License 


\title{
BIOMASS DERIVED ACTIVATED CARBON/EPOXY COMPOSITE AS MICROWAVE ABSORBING MATERIAL
}

\author{
Praveen Negi, Ashavani Kumar \\ Department of Physics, National Institute of Technology Kurukshetra, Haryana 136119, India \\ Email: ashavani@nitkkr.ac.in, ashavani@yahoo.com
}

\begin{abstract}
Herein, activated carbon/epoxy composite was synthesized, in which waste mango leaves biomass were used as carbon source. To create a sufficient amount of porosity in the biomass, a simple process of carbonization followed by its activation was used. The morphology and porosity of activated carbon material were studied by SEM, TEM images and BET analysis, which confirmed the presence of macro and mesopores in the material. The activated carbon/epoxy composite having $3 \mathrm{~mm}$ thickness gave the highest Reflection Loss (RL) of -39.57 $\mathrm{dB}$ at $6.48 \mathrm{GHz}$. At a thickness of just $2 \mathrm{~mm}$ the maximum effective absorption bandwidth (RL < $-10 \mathrm{~dB}$ ) of $2.14 \mathrm{GHz}$ was achieved. This excellent microwave absorption performance was attributed to large surface area due to porous and bowl type structures, which resulted in high impedance matching and subsequent attenuation of microwaves by interfacial and dipolar polarization. Present study provides porous activated carbon/epoxy composite that is cost effective, lightweight and has high structural stability for microwave absorption applications.
\end{abstract}

KEYWORDS: Activated carbon, microwave absorbing material, biomass, composite materials, reflection loss. 


\section{INTRODUCTION}

The rapid advancement in the area of electronics, communications, radar systems as well as increasing dependence on wireless technologies has resulted in an ever increasing electromagnetic pollution [1,2]. Therefore, the development of an efficient Microwave Absorbing Material (MAM) is in high demand. A high performance microwave absorbing material must display the properties like wide Effective Absorption Bandwidth (EAB), thin thickness, lightweight, high stability and low cost. Conventional MAM like ferrites and metal powders face drawbacks like heavyweight, poor chemical stability and require thick coating [1,3]. Recently carbon based MAM like carbon nanotubes [2,4-6], graphene [7-11] and graphene foam [12-14] has attracted attention because of their excellent properties like lightweight, high specific surface area, corrosion resistance and thermal stability [1,15], but due to high processing cost and complicated manufacturing techniques have limited their applications.

Recent studies have shown that biomass based porous carbon has the potential to become an efficient microwave absorbing material [1,3,15-19]. Most of the biomasses naturally possess network of microscale porous vascular bundles for water and ion transport, these built-in micropores contribute as an effective media in the composite, and improves the impedance matching and MW absorbing property of an absorber [1]. The porous materials facilitate multiple reflections of microwaves, which increases its path of propagation, leading to microwave energy loss inside the absorber material [3,20]. The solid-air interface formed due to porosity enhances the interfacial polarization hence attenuating a larger percentage of microwave. The porosity also helps to reduce the permittivity of the material which leads to better impedance matching [21]. Materials showing impedance close to air allow maximum amount of microwave to enter, hence reducing the direct reflection from the surface of the absorber material.

Apart from showing enhanced microwave absorbing properties, porous materials have low density which makes the composite lightweight. Therefore, biomass based porous materials have the potential to become efficient microwave material as they are cheap, lightweight, easy to scale and environment friendly. Our previous work has shown the excellent microwave absorption properties of waste mango leaves based activated carbon [16], it gave an excellent EAB of 5.17 $\mathrm{GHz}$ at thickness of just $1.75 \mathrm{~mm}$.

But most of the literature published on biomass based activated carbon for MAM application had used paraffin wax as their matrix. There is hardly any study for activated carbon/epoxy composite as MAM. Therefore, in the present work, the microwave absorption properties of waste mango leaves based activated carbon (AC)/epoxy composite is reported. The addition of epoxy to AC was done to provide structural strength and stability. Epoxy resins as a thermosetting matrix display excellent mechanical properties. The ease of processing, low cost and good chemical resistance of epoxy resins makes it a promising candidate for producing structurally strong composite material [22]. In the present study the AC/epoxy composite sample exhibited a maximum Reflection Loss (RL) of $-39.57 \mathrm{~dB}$ at a thickness of $3 \mathrm{~mm}$. 


\section{EXPERIMENTAL SECTION}

\subsection{Materials}

Mango leaves were accumulated from Institute premises, potassium hydroxide (KOH) was purchased from Merck life science Pvt. Ltd., Epoxy resin Huntsman Araldite LY 5052 and Hardener Huntsman LY Aradur 5052 were used as a matrix, and hydrochloric acid 35.4 wt\% (HCl) AR grade was purchased from Loba Chemie Pvt. Ltd.

\subsection{Synthesis of Activated Carbon}

The mango leaves were first washed with tap water and then with distilled water to remove dirt. After washing, the leaves were naturally dried and then grinded into powder. The $15 \mathrm{~g}$ of grinded powder was then pyrolyzed at $800{ }^{\circ} \mathrm{C}$ for 1 hour in a vacuum tubular furnace with a ramp rate of $5{ }^{\circ} \mathrm{C} / \mathrm{min}$ in nitrogen atmosphere. The resulting carbonized powder was thoroughly mixed with $\mathrm{KOH}$ (w/w 1:3) using mortar and pestle. The mixture was then put into a vacuum tubular furnace at $800{ }^{\circ} \mathrm{C}$ for 2 hours in nitrogen atmosphere for activation of carbonized mango leaves. The as prepared sample after activation was then washed with $\mathrm{HCl}(1: 1)$ solution followed by distilled water using centrifuge, till $\mathrm{pH}$ becomes neutral. The sample was then dried in oven and named as Activated Carbon (AC). The activation process using $\mathrm{KOH}$ resulted in defects and porosity in AC. The AC and epoxy composite was then prepared by mechanically mixing AC (40 wt \%) with epoxy resin (44 wt \%) and hardener (16 wt\%), the mixture was then pressed into toroidal shape $\left(\varphi_{\text {out }}=7.0 \mathrm{~mm}\right.$, $\varphi_{\text {in }}=3.0 \mathrm{~mm}$ ) and cured at $100{ }^{\circ} \mathrm{C}$ for 4 hours. The resulting final composite was named as Activated Carbon Epoxy (ACE).

\subsection{Characterizations}

Powder X-ray Diffraction (XRD) of AC was done by Rigaku Miniflex II with $\mathrm{Cu} \mathrm{K} \alpha$ radiation $(\lambda=1.54 \AA)$. Raman spectrum was obtained using Renishaw inVia Raman Microscope with laser excitation at $514 \mathrm{~nm}$. Morphology of AC was studies using images of Scanning Electron Microscopy (SEM, JEOL JSM-6390LV) and High Resolution Transmission Electron Microscope (HRTEM, FEI Tecnai G2 20 S-TWIN). Nitrogen adsorption-desorption isotherm was measured on Quantachrome Nova 2200e at 77.3 K. Multiple points Brunauer-EmmettTeller (BET) and Barrett, Joyner, Halenda $(\mathrm{BJH})$ methods were used to calculate specific surface area and pore size distribution respectively.

\subsection{Microwave absorption measurements}

The microwave absorption properties of sample ACE were measured at $2 \mathrm{~mm}, 3 \mathrm{~mm}$ and $4 \mathrm{~mm}$ thicknesses. The relative complex permittivity $\left(\varepsilon_{r}\right)$ and permeability $\left(\mu_{r}\right)$ measurement values were obtained by Agilent E8364B PNA series Vector Network Analyzer (VNA) in the frequency range of 2 to $18 \mathrm{GHz}$ using the Nicolson-Ross-Weir (NRW) algorithm [17]. The Reflection loss values were obtained using the metal backed values of $\mathrm{S}_{11}$. 


\section{RESULTS AND DISCUSSIONS}

\subsection{XRD and Raman analysis}

Fig. 1(a) represents the XRD pattern of the AC. The broad peak at $2 \theta=23.4^{\circ}$ corresponds to (002) plane and a minor peak at $2 \theta=43.2^{\circ}$ corresponds to (100) plane. These planes confirms the graphitic carbon nature of $\mathrm{AC}[3,16]$.

Raman spectra of AC as shown in Fig. 1(b) displays a typical D band at $1359 \mathrm{~cm}^{-1}$ and $\mathrm{G}$ band at $1597 \mathrm{~cm}^{-1}$, these two bands are the characteristic signature of graphitic materials. The $\mathrm{G}$ band is due to the $\mathrm{E}_{2 \mathrm{~g}}$ vibrational mode present within aromatic carbon rings and it also represents the degree of graphitization [23], whereas D band arises due to defects and disorder introduced into $\mathrm{sp}^{2}$ carbon. The intensity ratio value of the peaks $\mathrm{ID}_{\mathrm{D}} / \mathrm{IG}_{\mathrm{G}}=0.95$, which is proportional to the degree of graphitization of carbon material [18].
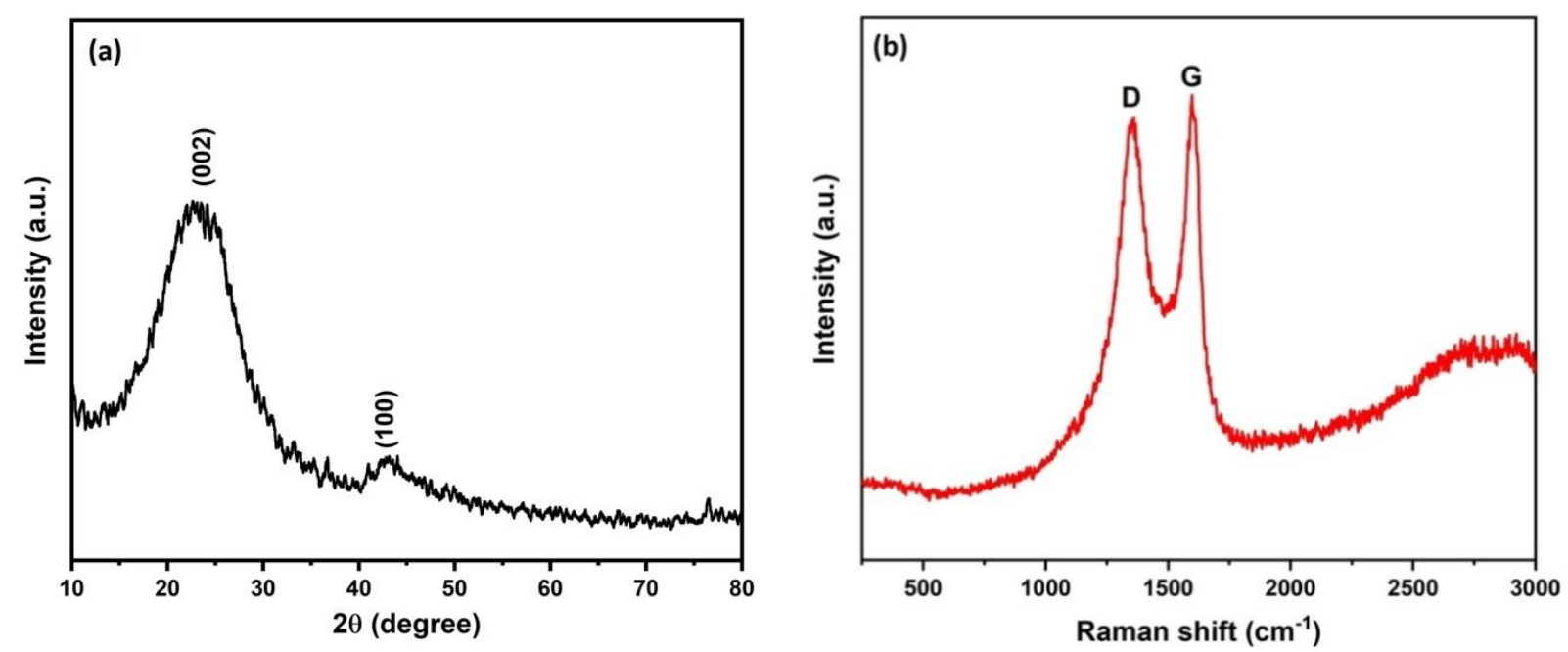

Fig. 1 (a) XRD pattern and (b) Raman spectrum of AC.

\subsection{BET and Morphological analysis}

Nitrogen adsorption-desorption isotherm of AC has already been discussed in our previous study [16]. In brief the sample gave type IV isotherm suggesting the presence of mesopores in the sample $[24,25]$. The pore size distribution calculated by BJH with total pore volume was 0.274 $\mathrm{cm}^{2} / \mathrm{g}$, it clearly shows that the pore size of all the pores are above $2 \mathrm{~nm}$ which confirms the formation of mesoporous in AC. The activation process by $\mathrm{KOH}$ resulted in a network of mesopores and macropores, which resulted in a large specific surface area $S_{\mathrm{BET}}=683.146 \mathrm{~m}^{2} / \mathrm{g}$. The morphology of AC was studied by SEM and HRTEM images. Fig. 2(a) clearly shows the presence of porous network formed within the sample due to the activation process, whereas Fig. 2(b) depicts a continuous bowl-shaped morphology which can be a result of partial porosity 
induced during activation process. Fig. 2(c,d) depicts the HRTEM images which confirms the presence of mesopores within the sample.
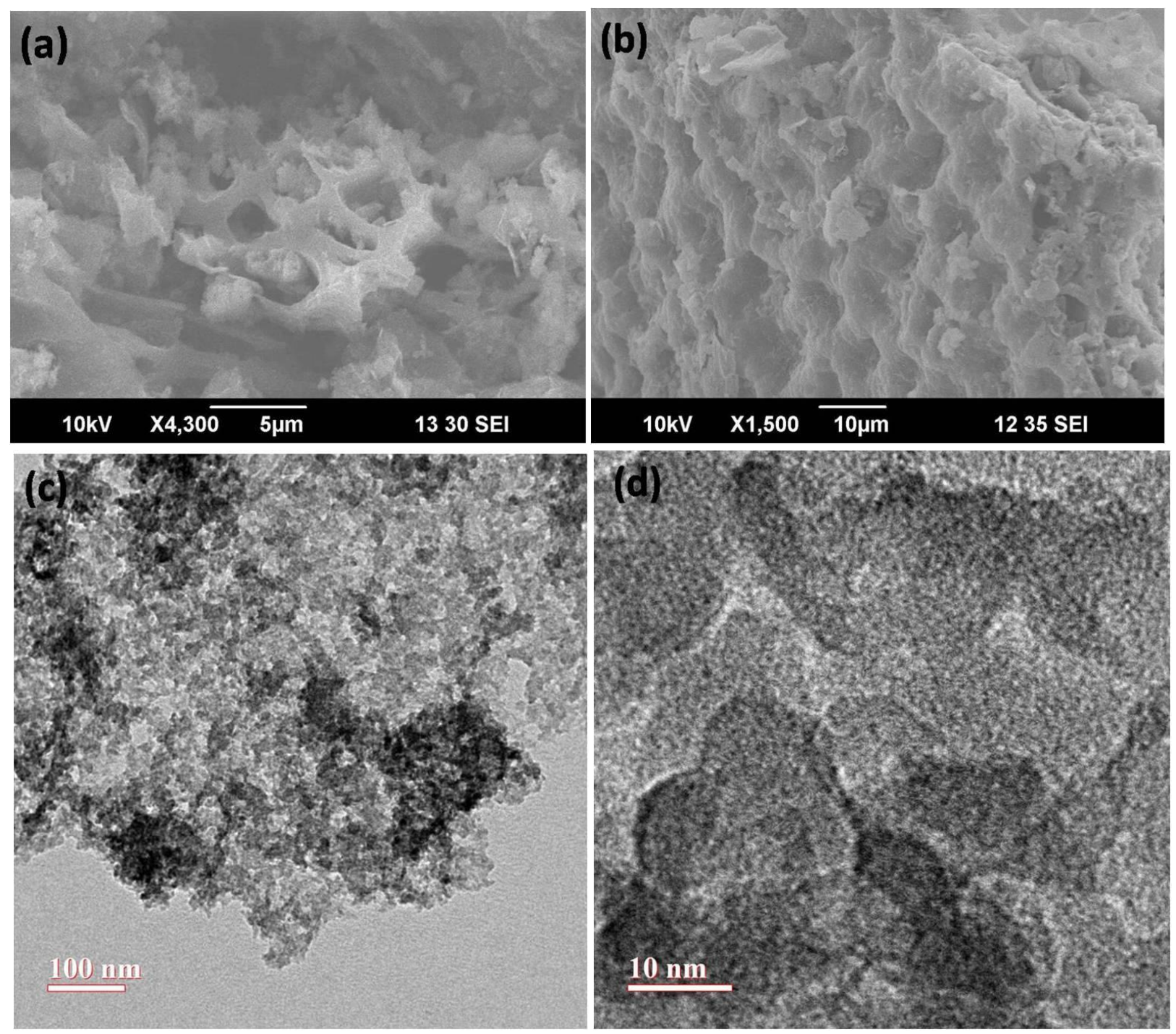

Fig. 2 (a, b) SEM and (c, d) TEM images of AC.

\subsection{Microwave Absorption Properties}

The microwave absorption properties of an absorber are mainly studied by two important parameters that are complex permittivity $\left(\varepsilon_{r}=\varepsilon^{\prime}-j \varepsilon^{\prime \prime}\right)$ and complex permeability $\left(\mu_{r}=\right.$ $\left.\mu^{\prime}-j \mu^{\prime \prime}\right)$. Where the real parts $\left(\varepsilon^{\prime}\right.$ and $\left.\mu^{\prime}\right)$ corresponds to energy storage and imaginary parts $\left(\varepsilon\right.$ "and $\left.\mu^{\prime \prime}\right)$ corresponds to energy dissipation [17,21]. The ACE is non-magnetic therefore the values of $\mu_{r}$ were taken to be 1 , hence the microwave absorption performance shown by ACE was solely because of dielectric part. Fig. 3(a) represents the relation of $\varepsilon^{\prime}$ and $\varepsilon^{\prime \prime}$ with frequency. 
The $\varepsilon^{\prime}$ value for the sample at initial frequency range remains nearly constant till $8.6 \mathrm{GHz}$ with minor variations, reaching a maximum value of 17.2 at $6.8 \mathrm{GHz}$.
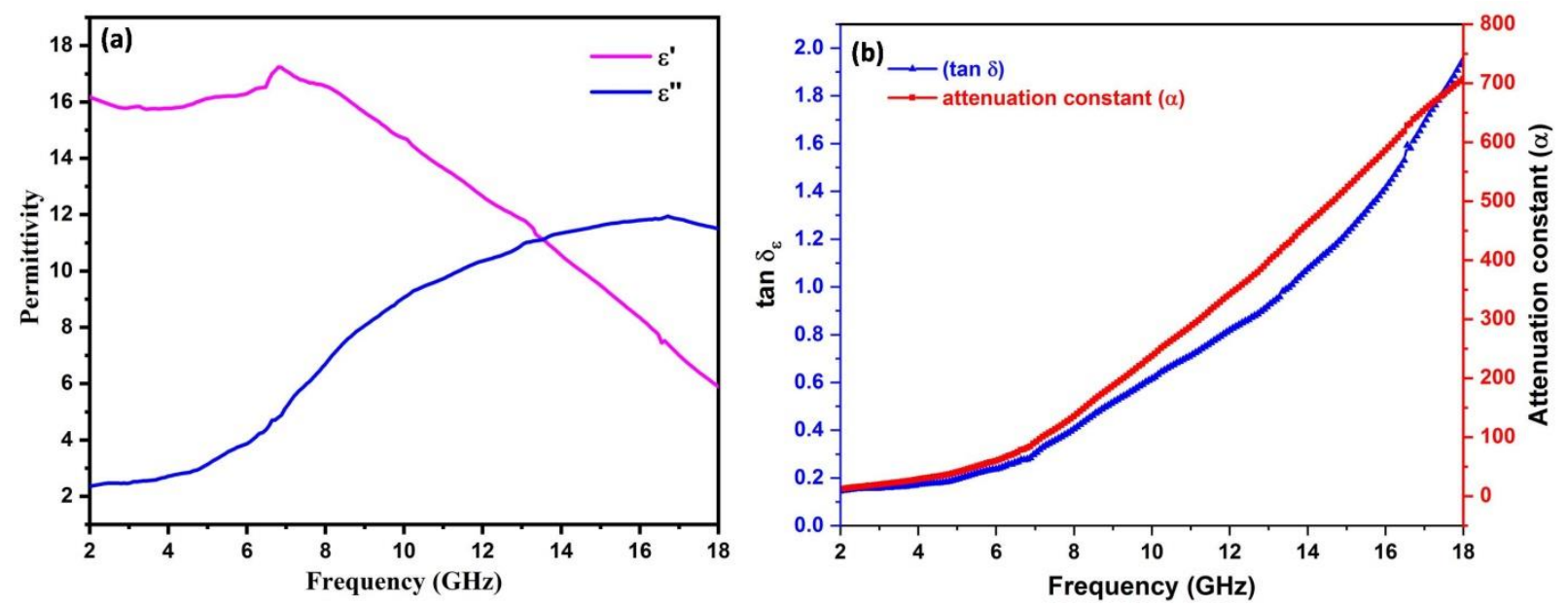

Fig. 3 Frequency dependence of (a) Relative permittivity, (b) Dielectric loss tangent and attenuation constant for ACE.

But thereafter, $\varepsilon^{\prime}$ value decreases with increasing frequency. Whereas $\varepsilon^{\prime \prime}$ value increases with increasing frequency reaching a maximum value of 11.9 at $16.7 \mathrm{GHz}$ and then shows a decreasing trend. The large values of $\varepsilon^{\prime}$ maybe attributed to the interfacial polarization and dipolar polarization phenomenon [19], the porous structure of AC results in the increase in surface area which facilitates the accumulation of electrons at the pore-solid interfaces [3], resulting in enhanced interfacial polarisation of microwaves [15].

In order to study the materials microwave energy loss ability, dielectric loss tangent $[15,26]$ and attenuation constant [27,28] were calculated using the equation 2 and 3 respectively:

$$
\begin{gathered}
\tan \delta_{\varepsilon}=\varepsilon^{\prime \prime} / \varepsilon^{\prime} \\
\alpha=\frac{\pi \mathrm{f}}{c}\left(2\left(\mu^{\prime \prime} \epsilon^{\prime \prime}-\mu^{\prime} \epsilon^{\prime}+\left(\left(\mu^{\prime \prime 2}+\mu^{\prime 2}\right)\left(\epsilon^{\prime \prime 2}+\epsilon^{\prime 2}\right)\right)^{1 / 2}\right)\right)^{1 / 2}
\end{gathered}
$$

Fig. 3(b) depicts the relation of dielectric loss $\left(\tan \delta_{\varepsilon}\right)$ and attenuation constant $(\alpha)$ of ACE with frequency. The value of $\tan \delta_{\varepsilon}$ as well as of $\alpha$ increases with increase in frequency, reaching a maximum value of 1.95 and 707 at $18 \mathrm{GHz}$ respectively.

Fig. 4(b) depicts impedance matching (Z) curve for ACE at different thicknesses, which is an important parameter that effects the performance of an absorber $[2,17,20]$, and represented as:

$$
Z=\left|Z_{\text {in }} / Z_{o}\right|=\left|\sqrt{\mu_{r} / \varepsilon_{r}} \tanh \left[j \frac{2 \pi f d}{c} \sqrt{\left(\mu_{r} \varepsilon_{r}\right)}\right]\right|
$$


Where $Z_{\text {in }}$ is the input impedance of an absorber, $Z_{0}$ is the impedance of free space, c is the speed of light, $f$ is the frequency of the electromagnetic waves and $\mathrm{d}$ is the thickness of the absorber.

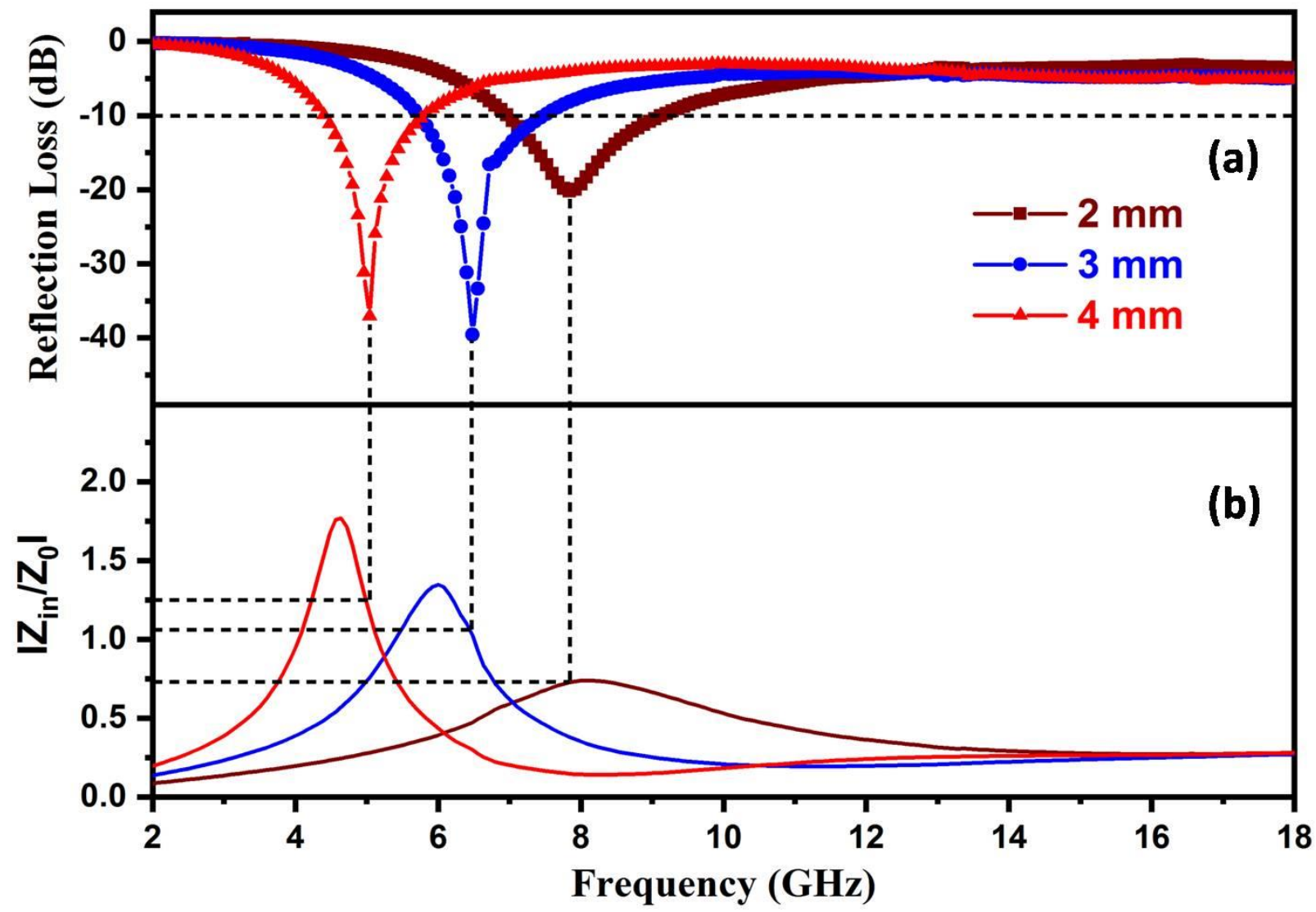

Fig. 4 Frequency dependence of (a) Reflection loss and (a) $\left|Z_{\text {in }} / Z_{o}\right|$ for ACE.

Closer the value of $\mathrm{Z}$ to 1 means better impedance matching, which allows more amount of microwave to enter into an absorber contributing in better microwave absorbing performance.

Fig. 4(a) shows the RL curves of ACE sample at different thicknesses. At $2 \mathrm{~mm}$ thickness a maximum EAB of $2.14 \mathrm{GHz}$ was achieved. Whereas the highest reflection loss of $-39.57 \mathrm{~dB}$ at $6.48 \mathrm{GHz}$ was obtained at $3 \mathrm{~mm}$ thickness. By comparing Fig. 4(a) and Fig. 4(b) it is clearly evident that the maximum RL for ACE at different thicknesses was achieved when there is maximum impedance matching.

This enhanced performance by ACE can be attributed to high impedance matching allowing more microwaves to enter into absorber and then subsequent attenuation of microwaves by interfacial polarization and dipolar polarization. 


\section{CONCLUSIONS}

Waste biomass (mango leaves) derived Activated Carbon and Epoxy composite (ACE) was synthesized as a microwave absorbing material. The porosity generated by $\mathrm{KOH}$ activation process provided large surface area $\left(\mathrm{S}_{\mathrm{BET}}\right)=683.146 \mathrm{~m}^{2} / \mathrm{g}$, which facilitated high impedance matching and attenuation of microwaves. The highest reflection loss of $-39.57 \mathrm{~dB}$ was obtained at $3 \mathrm{~mm}$ thickness and a maximum EAB of $2.14 \mathrm{GHz}$ was achieved at $2 \mathrm{~mm}$ thickness. Thus, activated carbon/epoxy composite is a porous, cost-effective, lightweight composite material and has the potential as microwave absorbing material.

\section{ACKNOWLEDGMENTS}

Author Praveen Negi is thankful to the Director, National Institute of Technology Kurukshetra for providing Institute fellowship. The author is also thankful to Materials Research Centre (MRC) MNIT Jaipur for performing HRTEM characterization. The authours are also thankful to the Director, DMSRDE, DRDO Kanpur for VNA characterization.

\section{REFERENCES}

[1] L. Liu, S. Yang, H. Hu, T. Zhang, Y. Yuan, Y. Li, X. He, Lightweight and Efficient Microwave-Absorbing Materials Based on Loofah-Sponge-Derived Hierarchically Porous Carbons, ACS Sustain. Chem. Eng. 7 (2019) 1228-1238.

https://doi.org/10.1021/acssuschemeng.8b04907.

[2] N. Li, G.W. Huang, Y.Q. Li, H.M. Xiao, Q.P. Feng, N. Hu, S.Y. Fu, Enhanced microwave absorption performance of coated carbon nanotubes by optimizing the $\mathrm{Fe} 3 \mathrm{O} 4$ nanocoating structure, ACS Appl. Mater. Interfaces. 9 (2017) 2973-2983.

https://doi.org/10.1021/acsami.6b13142.

[3] X. Qiu, L. Wang, H. Zhu, Y. Guan, Q. Zhang, Lightweight and efficient microwave absorbing materials based on walnut shell-derived nano-porous carbon, Nanoscale. 9 (2017) 7408-7418. https://doi.org/10.1039/c7nr02628e.

[4] F. Wen, F. Zhang, Z. Liu, Investigation on microwave absorption properties for multiwalled carbon nanotubes/Fe/Co/Ni nanopowders as lightweight absorbers, J. Phys. Chem. C. 115 (2011) 14025-14030. https://doi.org/10.1021/jp202078p.

[5] B.P. Singh, Prasanta, V. Choudhary, P. Saini, S. Pande, V.N. Singh, R.B. Mathur, Enhanced microwave shielding and mechanical properties of high loading MWCNTepoxy composites, J. Nanoparticle Res. 15 (2013). https://doi.org/10.1007/s11051-0131554-0.

[6] L. Deng, M. Han, Microwave absorbing performances of multiwalled carbon nanotube composites with negative permeability, Appl. Phys. Lett. 91 (2007) 2005-2008. https://doi.org/10.1063/1.2755875.

[7] P. Liu, Z. Yao, J. Zhou, Z. Yang, L.B. Kong, Small magnetic Co-doped NiZn ferrite/graphene nanocomposites and their dual-region microwave absorption 
performance, J. Mater. Chem. C. 4 (2016) 9738-9749.

https://doi.org/10.1039/c6tc03518c.

[8] M. Fu, Q. Jiao, Y. Zhao, H. Li, Vapor diffusion synthesis of CoFe2O4 hollow sphere/graphene composites as absorbing materials, J. Mater. Chem. A. 2 (2014) 735-744. https://doi.org/10.1039/c3ta14050d.

[9] J. Feng, Y. Hou, Y. Wang, L. Li, Synthesis of Hierarchical ZnFe2O4@SiO2@RGO CoreShell Microspheres for Enhanced Electromagnetic Wave Absorption, ACS Appl. Mater. Interfaces. 9 (2017) 14103-14111. https://doi.org/10.1021/acsami.7b03330.

[10] R. Shu, W. Li, X. Zhou, D. Tian, G. Zhang, Y. Gan, J. Shi, J. He, Facile preparation and microwave absorption properties of RGO/MWCNTs/ZnFe2O4 hybrid nanocomposites, J. Alloys Compd. 743 (2018) 163-174. https://doi.org/10.1016/j.jallcom.2018.02.016.

[11] C. Wang, X. Han, P. Xu, X. Zhang, Y. Du, S. Hu, J. Wang, X. Wang, The electromagnetic property of chemically reduced graphene oxide and its application as microwave absorbing material, Appl. Phys. Lett. 98 (2011) 2011-2014.

https://doi.org/10.1063/1.3555436.

[12] W. Ma, H. Chen, S. Hou, Z. Huang, Y. Huang, S. Xu, F. Fan, Y. Chen, Compressible Highly Stable 3D Porous MXene/GO Foam with a Tunable High-Performance Stealth Property in the Terahertz Band, ACS Appl. Mater. Interfaces. 11 (2019) 25369-25377. https://doi.org/10.1021/acsami.9b03406.

[13] P. Liu, Y. Zhang, J. Yan, Y. Huang, L. Xia, Z. Guang, Synthesis of lightweight N-doped graphene foams with open reticular structure for high-efficiency electromagnetic wave absorption, Chem. Eng. J. 368 (2019) 285-298. https://doi.org/10.1016/j.cej.2019.02.193.

[14] Y. Zhang, Y. Huang, T. Zhang, H. Chang, P. Xiao, H. Chen, Z. Huang, Y. Chen, Broadband and tunable high-performance microwave absorption of an ultralight and highly compressible graphene foam, Adv. Mater. 27 (2015) 2049-2053. https://doi.org/10.1002/adma.201405788.

[15] Z. Wu, K. Tian, T. Huang, W. Hu, F. Xie, J. Wang, M. Su, L. Li, Hierarchically Porous Carbons Derived from Biomasses with Excellent Microwave Absorption Performance, ACS Appl. Mater. Interfaces. 10 (2018) 11108-11115. https://doi.org/10.1021/acsami.7b17264.

[16] P. Negi, A. Kumar, A.K. Dixit, S. Kumar, A. Kumar, D. Ph, Activated carbon derived from mango leaves as an enhanced microwave absorbing material, Sustain. Mater. Technol. 27 (2021) e00244. https://doi.org/10.1016/j.susmat.2020.e00244.

[17] J. Fang, Y. Shang, Z. Chen, W. Wei, Y. Hu, X. Yue, Z. Jiang, Rice husk-based hierarchically porous carbon and magnetic particles composites for highly efficient electromagnetic wave attenuation, J. Mater. Chem. C. 5 (2017) 4695-4705. https://doi.org/10.1039/c7tc00987a.

[18] L. Wang, P. Zhou, Y. Guo, J. Zhang, X. Qiu, Y. Guan, M. Yu, H. Zhu, Q. Zhang, The 
effect of $\mathrm{ZnCl} 2$ activation on microwave absorbing performance in walnut shell-derived nano-porous carbon, RSC Adv. 9 (2019) 9718-9728. https://doi.org/10.1039/c8ra09932d.

[19] Q. Liu, D. Zhang, T. Fan, Electromagnetic wave absorption properties of porous carbon/Co nanocomposites, Appl. Phys. Lett. 93 (2008).

https://doi.org/10.1063/1.2957035.

[20] J. Fang, T. Liu, Z. Chen, Y. Wang, W. Wei, X. Yue, Z. Jiang, A wormhole-like porous carbon/magnetic particles composite as an efficient broadband electromagnetic wave absorber, Nanoscale. 8 (2016) 8899-8909. https://doi.org/10.1039/c6nr01863g.

[21] H. Zhao, Y. Cheng, H. Lv, G. Ji, Y. Du, A novel hierarchically porous magnetic carbon derived from biomass for strong lightweight microwave absorption, Carbon N. Y. 142 (2019) 245-253. https://doi.org/10.1016/j.carbon.2018.10.027.

[22] B.P. Singh, K. Saini, V. Choudhary, S. Teotia, S. Pande, P. Saini, R.B. Mathur, Effect of length of carbon nanotubes on electromagnetic interference shielding and mechanical properties of their reinforced epoxy composites, J. Nanoparticle Res. 16 (2014). https://doi.org/10.1007/s11051-013-2161-9.

[23] V.C. Tung, M.J. Allen, Y. Yang, R.B. Kaner, High-throughput solution processing of large-scale graphene, Nat. Nanotechnol. 4 (2009) 25-29. https://doi.org/10.1038/nnano.2008.329.

[24] H. Zhao, Y. Cheng, J. Ma, Y. Zhang, G. Ji, Y. Du, A sustainable route from biomass cotton to construct lightweight and high-performance microwave absorber, 2018. https://doi.org/10.1016/j.cej.2018.01.151.

[25] I. Union, O.F. Pure, A. Chemistry, INTERNATIONAL UNION OF PURE COMMISSION ON COLLOID AND SURFACE CHEMISTRY INCLUDING CATALYSIS * REPORTING PHYSISORPTION DATA FOR GAS / SOLID SYSTEMS with Special Reference to the Determination of Surface Area and Porosity, 57 (1985) 603619.

[26] C. Zhou, S. Geng, X. Xu, T. Wang, L. Zhang, X. Tian, F. Yang, H. Yang, Y. Li, Lightweight hollow carbon nanospheres with tunable sizes towards enhancement in microwave absorption, Carbon N. Y. 108 (2016) 234-241. https://doi.org/10.1016/j.carbon.2016.07.015.

[27] B. Zhang, J. Wang, X. Su, H. Duan, H. Cai, J. Wang, S. Yang, S. Huo, Enhanced microwave absorption properties of epoxy composites containing graphene decorated with core-shell Fe3O4@polypyrrole nanoparticles, J. Mater. Sci. Mater. Electron. 28 (2017) 12122-12131. https://doi.org/10.1007/s10854-017-7026-z.

[28] F. Qin, C. Brosseau, A review and analysis of microwave absorption in polymer composites filled with carbonaceous particles, J. Appl. Phys. 111 (2012). https://doi.org/10.1063/1.3688435. 
Figures
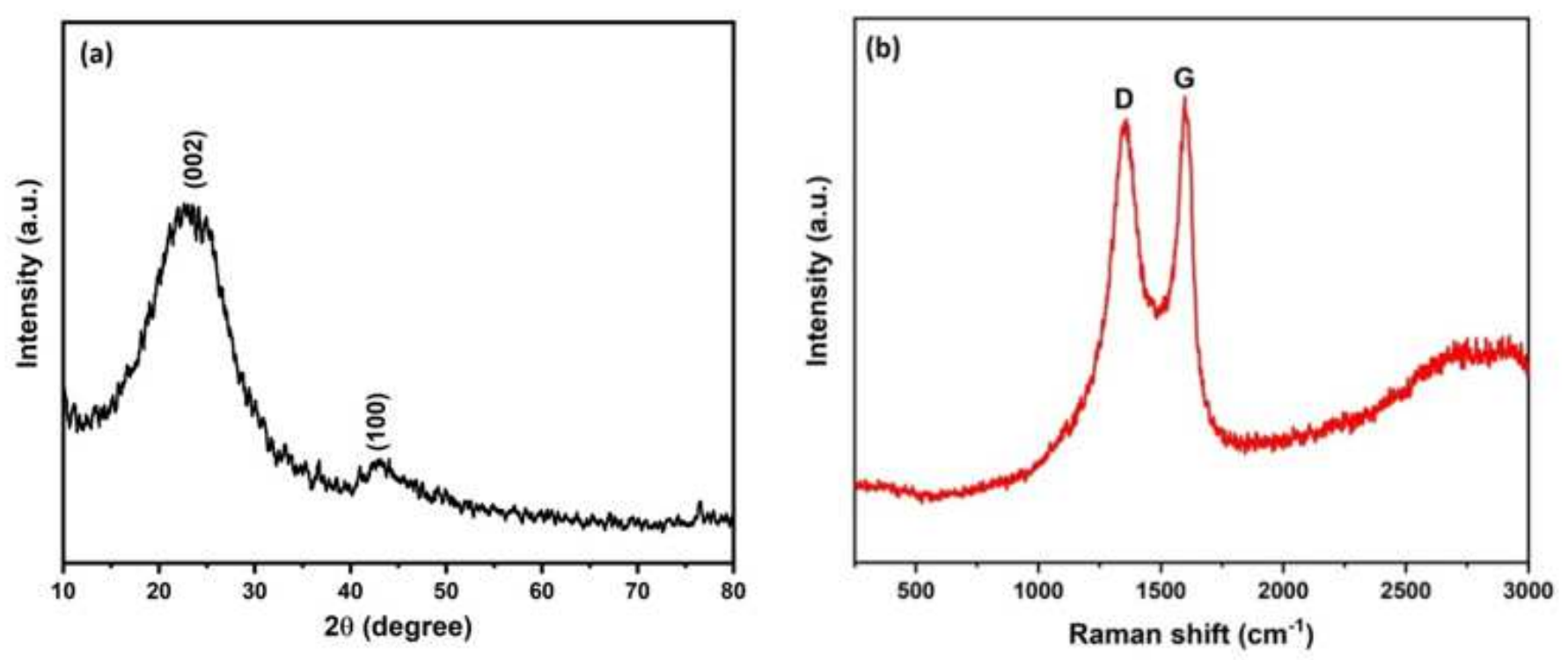

Figure 1

(a) XRD pattern and (b) Raman spectrum of AC. 

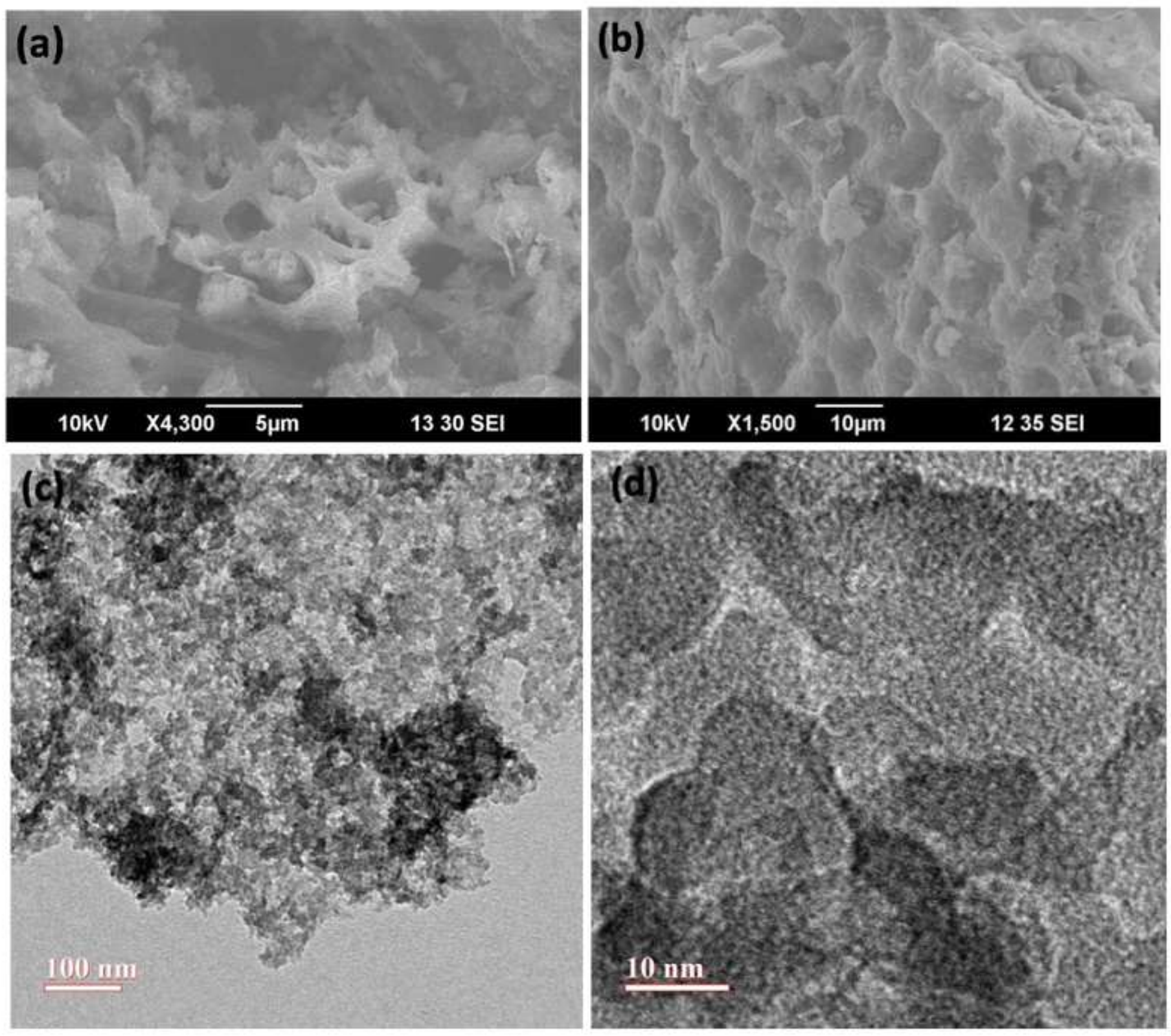

Figure 2

$(a, b)$ SEM and (c, d) TEM images of AC. 

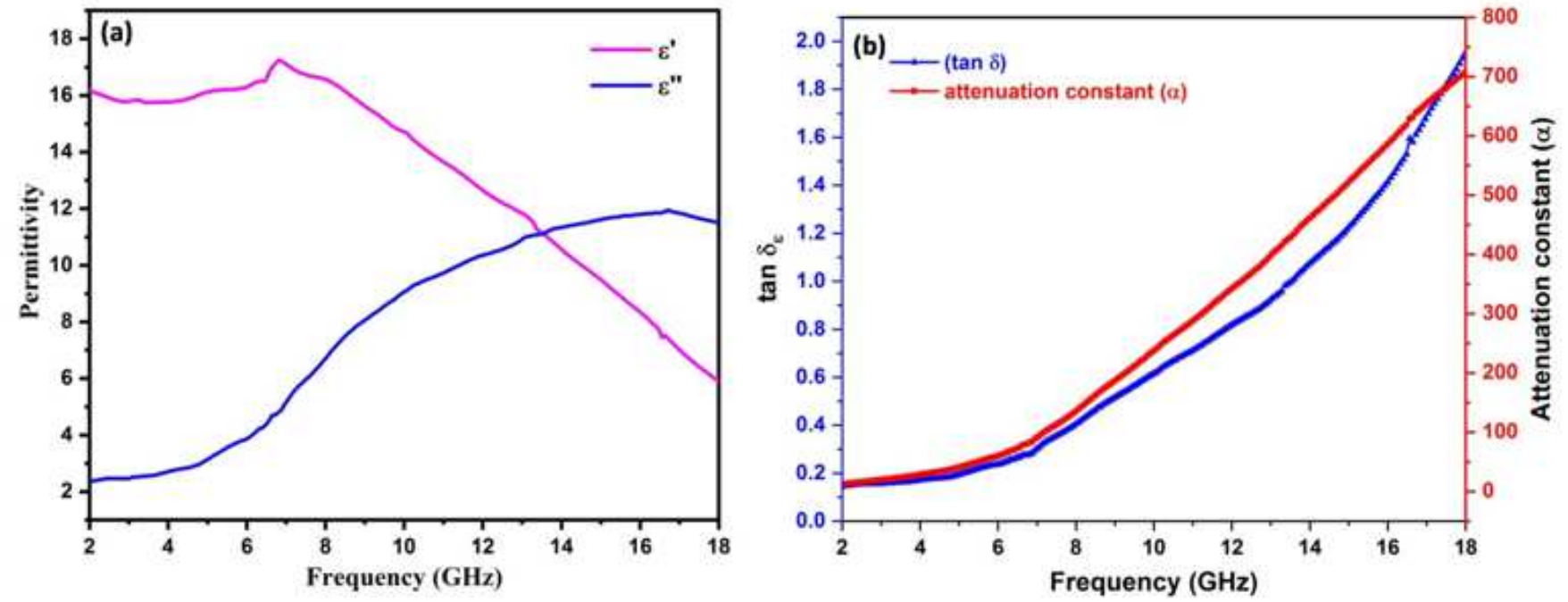

Figure 3

Frequency dependence of (a) Relative permittivity, (b) Dielectric loss tangent and attenuation constant for ACE.

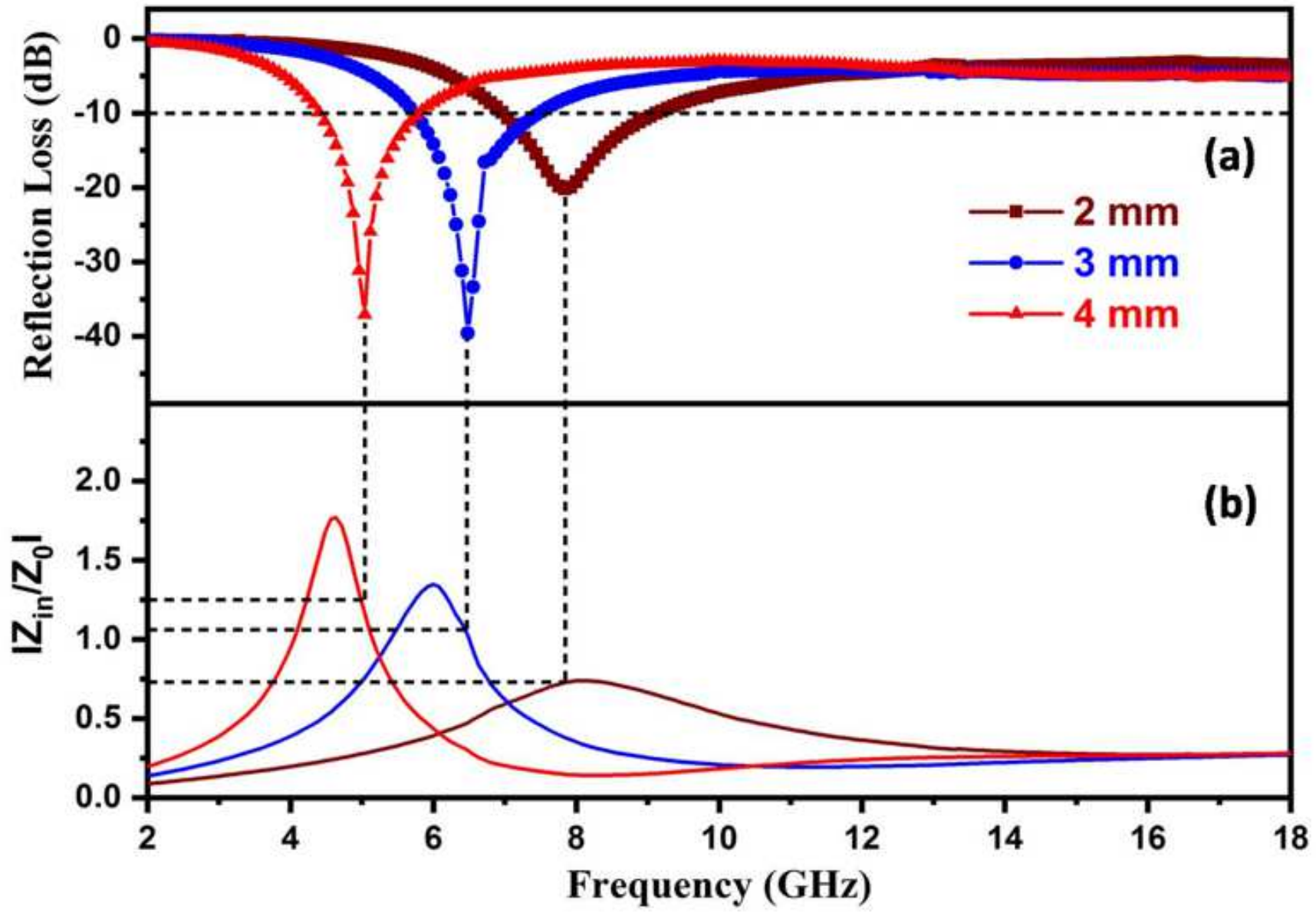

Figure 4 
Frequency dependence of (a) Reflection loss and (a) IZ_in/Z_o | for ACE. 